\title{
Klein Tunneling of Light in Fiber Bragg Gratings
}

\author{
Stefano Longhi \\ Dipartimento di Fisica, Politecnico di Milano, Piazza L. da Vinci 32, 20133 Milano, Italy \\ Correspondence should be addressed to Stefano Longhi, longhi@fisi.polimi.it
}

Received 28 May 2010; Accepted 29 July 2010

Academic Editor: Lorenzo Pavesi

Copyright (C) 2010 Stefano Longhi. This is an open access article distributed under the Creative Commons Attribution License, which permits unrestricted use, distribution, and reproduction in any medium, provided the original work is properly cited.

A photonic analogue of Klein tunneling (KT), that is, of the exotic property of relativistic electrons to pass a large repulsive and sharp potential step, is proposed for pulse propagation in a nonuniform fiber Bragg grating with an embedded chirped region. KT can be simply observed as the opening of a transmission window inside the grating stop band, provided that the impressed chirp is realized over a length of the order of the analogue of the Compton wavelength.

\section{Introduction}

A remarkable prediction of the Dirac equation is that a below-barrier electron can pass a large repulsive and sharp potential step without the exponential damping expected for a nonrelativistic particle. Such a transparency effect, originally predicted by Klein [1] and referred to as Klein tunneling (KT), arises from the existence of negative-energy solutions of the Dirac equation and requires a potential step height $\Delta V$ of the order of twice the rest energy $m c^{2}$ of the electron [2]. Relativistic tunneling across a smooth potential step, which describes the more physical situation of a constant electric field $E$ in a finite region of space of length $l$, was subsequently studied by Sauter [3]. Sauter showed that to observe barrier transparency, the potential increase $\Delta V \simeq$ $e E l$ should occur over a distance $l$ of the order or smaller than the Compton wavelength $\lambda_{C}=\hbar /(m c)$, the transmission probability rapidly decaying toward zero for a smoother potential increase [2-4]. The required field corresponds to the critical field for $e^{+} e^{-}$pair production in vacuum, and its value is extremely strong making the observation of relativistic KT for electrons very challenging. Therefore, growing efforts have been devoted to find experimentally accessible systems to investigate analogs of relativistic KT [5]. Recently, great interest has suscitated the proposal [6] and first experimental evidences $[7,8]$ of KT for non-relativistic electrons in graphene, which behave like massless Dirac fermions. On the other hand, optics has offered on many occasions a test bed to investigate the dynamical aspects embodied in a wide variety of coherent quantum phenomena (see, for instance, [9] and references therein). In optics, several proposals of KT analogs have been suggested as well, including light propagation in deformed honeycomb photonic lattices [10] whose band structure is similar to the one of graphene $[11,12]$, light refraction at the interface between positive-index and negative-index media [13], spatial light propagation in binary waveguide arrays [14], and stationary light pulses in an atomic ensemble with electromagnetically induced transparency [15]. The experimental implementations of such schemes, however, might be a nontrivial matter, and an experimental observation of KT for photons is still lacking. On the other hand, multilayer and Bragg dielectric structures, such as fiber Bragg gratings (FBGs), are rather simple photonic devices with flexible design that have been successfully demonstrated to provide an accessible laboratory tool to investigate photonic analogues of non-relativistic tunneling phenomena [16-18]. Here it is shown that an optical analogue of KT can be achieved in a nonuniform FBG composed by two periodic sections linked by a chirped section which mimics an external potential step in the Dirac equation. Such a FBG-based system might be considered the simplest system proposed so far in order to observe Klein tunneling in any optical system.

\section{Quantum-Optical Analogy}

The starting point of our analysis if provided by a standard model of light propagation in an FBG with a longitudinal 
refractive index $n\left(z^{\prime}\right)=n_{0}+\Delta n m\left(z^{\prime}\right) \cos \left[2 \pi z^{\prime} / \Lambda+2 \phi\left(z^{\prime}\right)\right]$, where $n_{0}$ is the effective mode index in absence of the grating, $\Delta n \ll n_{0}$ is the peak index change of the grating, $\Lambda$ is the nominal period of the grating defining the reference frequency $\omega_{B}=\pi c /\left(\Lambda n_{0}\right)$ of Bragg scattering, $c$ is the speed of light in vacuum, and $m\left(z^{\prime}\right), 2 \phi\left(z^{\prime}\right)$ describe the slow variation, as compared to the scale of $\Lambda$, of normalized amplitude and phase, respectively, of the index modulation. Note that the local spatial frequency of the grating is $k\left(z^{\prime}\right)=$ $2 \pi / \Lambda+2\left(d \phi / d z^{\prime}\right)$, so that the local chirp rate is $C=$ $d k / d z^{\prime}=2\left(d^{2} \phi / d z^{\prime 2}\right)$. The periodic index modulation leads to Bragg scattering between two counterpropagating waves at frequencies close to $\omega_{B}$. By letting $E\left(z^{\prime}, t\right)=$ $\varphi_{1}\left(z^{\prime}, t\right) \exp \left[-i \omega_{B} t+i k_{B} z^{\prime}+i \phi\left(z^{\prime}\right)\right]+\varphi_{2}\left(z^{\prime}, t\right) \exp \left[-i \omega_{B} t-\right.$ $\left.i k_{B} z^{\prime}-i \phi\left(z^{\prime}\right)\right]+c . c$. for the electric field in the fiber, where $k_{B}=\pi / \Lambda$, the envelopes $\varphi_{1}$ and $\varphi_{2}$ of counterpropagating waves satisfy the coupled-mode equations [19]

$$
\begin{array}{r}
i\left[\partial_{z^{\prime}}+\left(\frac{1}{v_{g}}\right) \partial_{t}\right] \varphi_{1}=\left(\frac{d \phi}{d z^{\prime}}\right) \varphi_{1}-\kappa\left(z^{\prime}\right) \varphi_{2}, \\
i\left[-\partial_{z^{\prime}}+\left(\frac{1}{v_{g}}\right) \partial_{t}\right] \varphi_{2}=\left(\frac{d \phi}{d z^{\prime}}\right) \varphi_{2}-\kappa\left(z^{\prime}\right) \varphi_{1},
\end{array}
$$

where $\kappa\left(z^{\prime}\right) \equiv\left[k_{B} m\left(z^{\prime}\right) \Delta n\right] /\left(2 n_{0}\right)$ and $v_{g} \sim c / n_{0}$ is the group velocity at the Bragg frequency. The analogy between pulse propagation in the FBG and the Dirac equation in presence of an electrostatic field is at best captured by introducing the dimensionless variables $z=z^{\prime} / Z$ and $\tau=t / T$, with characteristic spatial and time scales $Z=2 n_{0} /\left(k_{B} \Delta n\right)$ and $T=Z / v_{g}$, and the new envelopes $\psi_{1,2}\left(z^{\prime}\right)=\left[\varphi_{1}\left(z^{\prime}\right) \mp\right.$ $\left.\varphi_{2}\left(z^{\prime}\right)\right] / \sqrt{2}$. In this way, (1) can be cast in the Dirac form

$$
i \partial_{\tau} \psi=-i \sigma_{1} \partial_{z} \psi+m \sigma_{3} \psi+V(z) \psi
$$

for the spinor wave function $\psi=\left(\psi_{1}, \psi_{2}\right)^{T}$, where $V(z)=$ $(d \phi / d z)$ and $\sigma_{1,3}$ are the Pauli matrices, defined by

$$
\sigma_{1}=\left(\begin{array}{ll}
0 & 1 \\
1 & 0
\end{array}\right), \quad \sigma_{3}=\left(\begin{array}{cc}
1 & 0 \\
0 & -1
\end{array}\right) .
$$

In its present form, (2) is formally analogous to the onedimensional Dirac equation with $\hbar=c=1$ in presence of an external electrostatic potential $V(z), m$ playing the role of a dimensionless (and generally space-dependent) rest mass (see, for instance, $[2,4]$ ). As is wellknown, a nonvanishing mass $m$ is responsible for the existence of a forbidden energy region, which separates the positive- and negative-energy branches of the massive Dirac equation. The optical analogue of the forbidden energy region is precisely the photonic stop band of the periodic grating. As the refractive index modulation of the grating, that is, the mass term $m$ in the Dirac equation (2), is decreased, the stop band region shrinks and the limit of a massless Dirac equation (similar to the one describing the dynamics of electrons in graphene near a Dirac point) is attained. The additional external potential $V$ in (2), related to the chirp of the grating according to $V(z)=(d \phi / d z)$, changes the local position of the forbidden energy region. Therefore, pulse propagation in an FBG with a suitably designed chirp profile can be used to mimic the relativistic tunneling of a wave packet in a potential step $V(z)$. It should be noted that, as compared to other photonic analogues of KT recently proposed in [10, 14] and based on spatial light propagation in periodic photonic structures, the phenomenon of KT occurring in FBGs and discussed in the following section involves the temporal (rather than the spatial) light dynamics and can be therefore simply investigated in the frequency domain by spectrally resolved transmission measurements.

\section{Klein Tunneling}

To realize the analogue of $\mathrm{KT}$, let us first assume that the optical pulse propagates in a region of the grating where $m(z)$ is uniform and equal to one, and let us assume a chirp profile that mimics a step potential with an increase from $V=0$ to $V=\Delta V$ which occurs over a length $l$ (see Figure 1). Since for the Dirac equation (2) written in dimensionless units the Compton length is $\lambda_{C}=1$ and the rest energy is $m c^{2}=1$; according to Sauter's analysis KT is expected to be observable for $l$ smaller than $\sim 1$ and for a potential height $\Delta V$ larger than $2[2-4]$. The process of $\mathrm{KT}$ and tunneling inhibition for a smooth potential step can be simply explained by a graphical analysis of the space-energy diagrams $(z, \Omega)$ of the one-dimensional Dirac equation [2], which are shown in Figures 1(a) and 1(b) for a sharp and for a smooth potential step, respectively. For the sake of clearness, in the figures the potential $V(z)$ has been chosen to yield a nonvanishing and constant chirp rate over a length $l$; different forms for the potential step, such as the profile $V(z)=(\Delta V / 2)[1+\tanh (z / l)]$ considered in the seminal work by Sauter [3], can be assumed as well without changing the main results.

The space-energy diagrams of Figures $1(\mathrm{a})$ and 1(b) schematically show the behavior of the energy spectrum of (2) versus $z$, which is composed by two branchesthe electron and positron energy branches of the Dirac equation-separated by a gap of width $2 m(z)$ and centered along the curve $\Omega=V(z)$. The gap regions are visualized in the diagrams by the shaded areas. A wave packet (optical pulse) in the electron branch with an initial mean energy $\Omega_{0}\left(1<\Omega_{0}<\Delta V-1\right)$ coming from $z \rightarrow-\infty$ tunnels into the $z>0$ region after crossing a forbidden energy region, indicated by the bold segment $A B$ in Figure 1(b). The forbidden gap region $A B$ vanishes for a sharp potential step $(l=0$, see Figure $1(\mathrm{a}))$. According to Sauter's analysis $[2,3]$, the tunneling probability is appreciable provided that $l$ is smaller than $\sim 1$. In the FBG context, the energy diagrams of Figure 1 are equivalent to the band-reflection diagrams introduced by Poladian for a graphical analysis of nonuniform gratings [20], where the energy $\Omega$ represents the frequency detuning of the incoming wave from the Bragg frequency $\omega_{B}$. The Sauter's condition $l<\sim 1$ for KT can be derived following the analysis of [20] by computation of the transmittance of the effective grating associated to the evanescent region $\mathrm{AB}$ shown in Figure 1(b) (see Section 5.1 


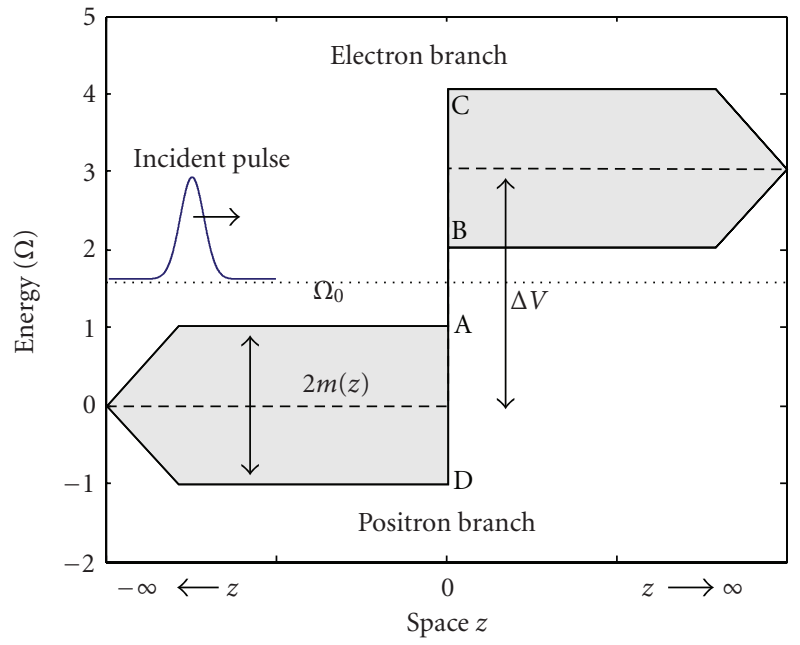

(a)

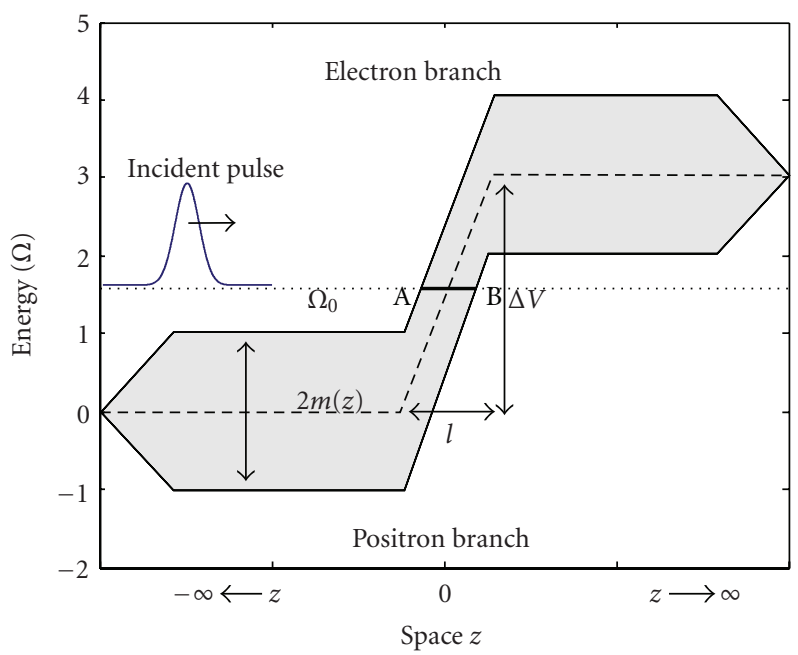

(b)

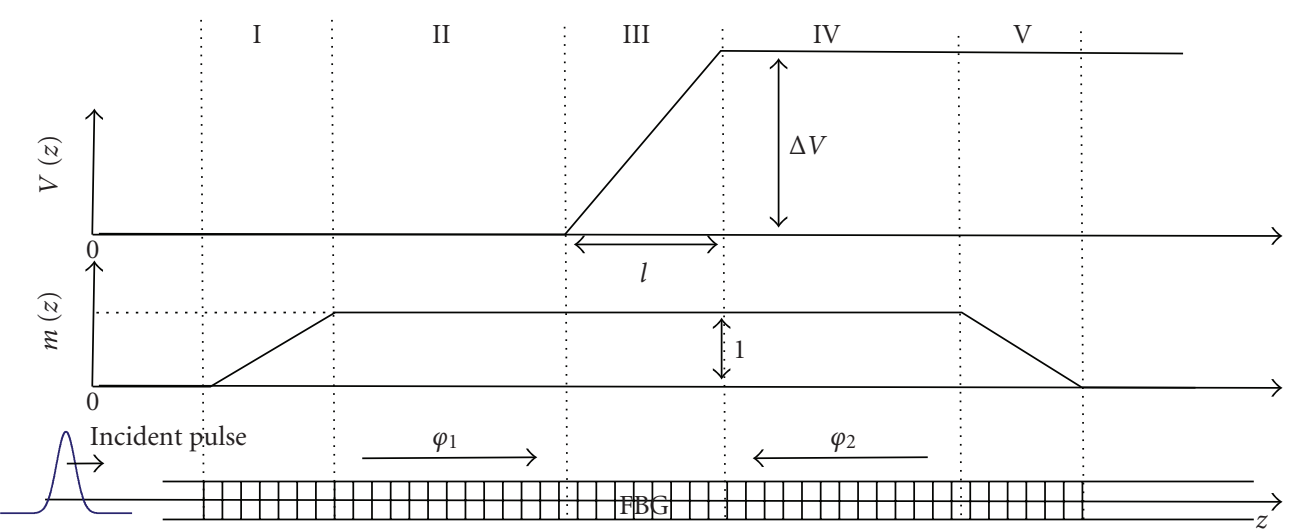

(c)

FIgURE 1: Energy diagrams of the Dirac equation (2) for (a) a sharp and (b) a smooth potential step $V(z)$ of height $\Delta V$. The shaded regions are the forbidden energies that separate the electron and positron states, the dotted horizontal line is the energy $\Omega_{0}$ of the incoming wave packet, and the dashed curve is the shape of the potential step $V(z)$. (c) Schematic of the grating structure that realizes the optical analogue of relativistic tunneling across a potential step. The grating comprises five sections, denoted by roman numbers (from I to V) that are defined by the amplitude $m(z)$ and phase gradient $V(z)=(d \phi / d z)$ grating profiles.

of [20]). In the previous discussion, we assumed $m(z)=$ 1 , however for a grating with finite spatial extent one has $m(z) \rightarrow 0$ as $z \rightarrow \pm \infty$. To inject and to eject the optical pulse into the $m(z)=1$ grating region around $z=0$, an input and an output apodization sections can be introduced, which adiabatically convert the input and output wave packets from the $m(z)=0$ regions into the $m(z)=1$ grating region (see Figure 1). Therefore, the general structure of the FBG that realizes a photonic analogue of relativistic tunneling across a potential step consists of five sections, as shown in Figure 1(c): two boundary apodization sections (regions I and V), and two uniform sections (regions II and IV) separated by a central chirped section of length $\sim l$ (region III). In Figures 2(a)-2(c) typical examples of pulse tunneling across the potential step $V(z)=(\Delta V / 2)[1+$ $\tanh (z / l)]$ are presented, showing KT for a sharp potential step (Figure 2(a)) and inhibition of tunneling as the step gets smooth (Figures 2(b) and 2(c)). The figures depict the temporal evolution of $\left|\psi_{1}\right|^{2}+\left|\psi_{2}\right|^{2}=\left|\varphi_{1}\right|^{2}+\left|\varphi_{2}\right|^{2}$-which is proportional to the field intensity averaged in time over a few optical cycles and in space over a few wavelengths-as obtained by numerical analysis of (1) for a grating length of $z=160$ with a quarter-cosine apodization profile (see Figure 2(d)), $\Delta V=6$, and for a few values of $l$. A forwardpropagating Gaussian pulse $\varphi_{1}$ of mean energy $\Omega_{0}=2$ coming from $z \rightarrow-\infty$ and of duration (FWHM in intensity) $\tau_{p}=5$ has been assumed as an initial condition. For typical parameter values $n_{0}=1.45, \Delta n=3.3 \times 10^{-4}$, and $\lambda_{B} \equiv$ $2 n_{0} \Lambda=1560 \mathrm{~nm}$, which apply to FBGs used in optical communications, the spatial and temporal scales in Figure 2 are $Z \simeq 1.5 \mathrm{~mm}$ and $T \simeq 7.3 \mathrm{ps}$, respectively. Hence, in physical units the grating length is $L \simeq 24 \mathrm{~cm}$ whereas the optical analogue of the Compton length is $\lambda_{C}=Z \simeq 1.5 \mathrm{~mm}$. Such nonuniform FBG structures should be realizable with current FBG technology based on UV continuous laser writing [21]. It should be finally noticed that, as in an 


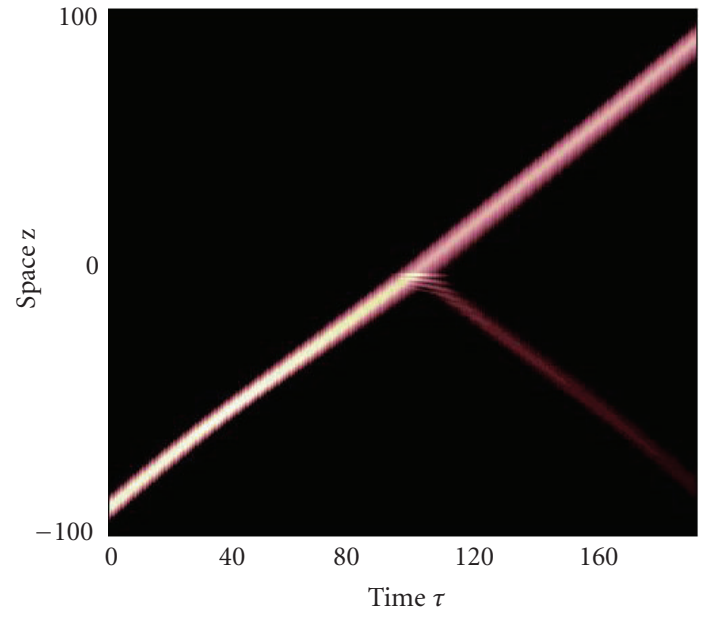

(a)

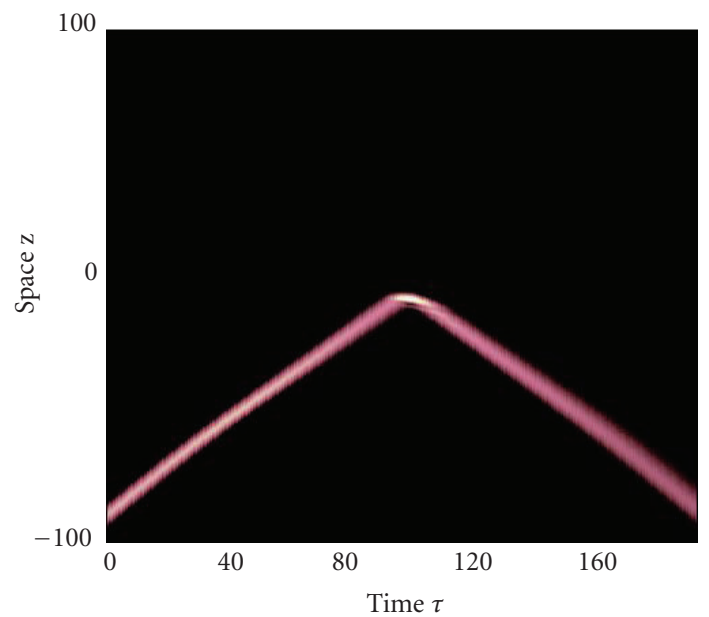

(c)

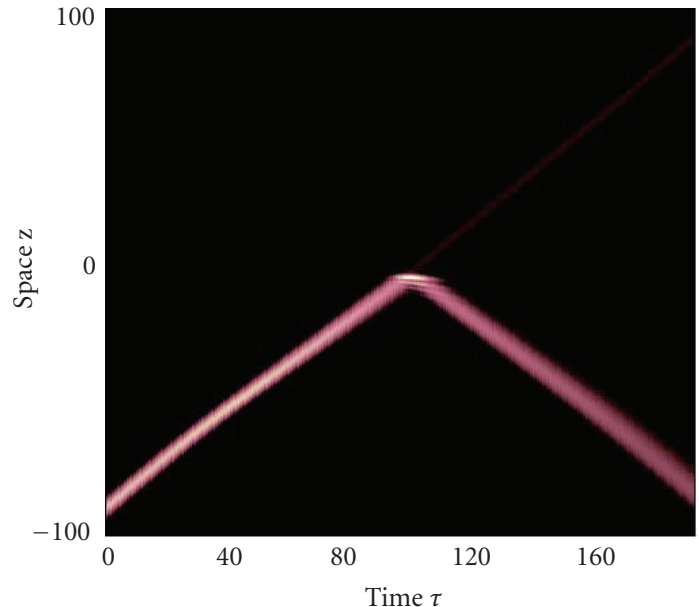

(b)

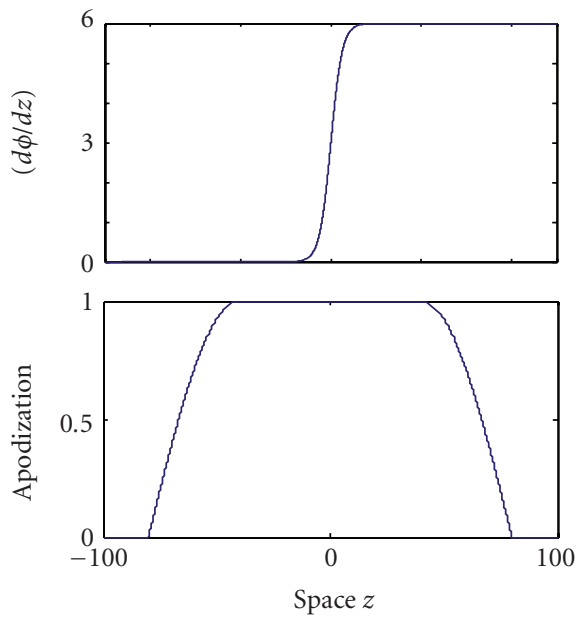

(d)

FIgure 2: (a)-(c) Pulse propagation in a FBG with an chirp profile $V(z)=(\Delta V / 2)[1+\tanh (z / l)]$ for $\Delta V=6$ and for (a) $l=0.1$, (b) $l=1.5$, and (c) $l=5$. (d) Profiles of $V=(d \phi / d z)$ (upper plot) and of grating amplitude $m(z)$ (apodization profile, lower plot). The potential $V$ is shown for $l=5$, corresponding to the simulation of Figure 2(c).

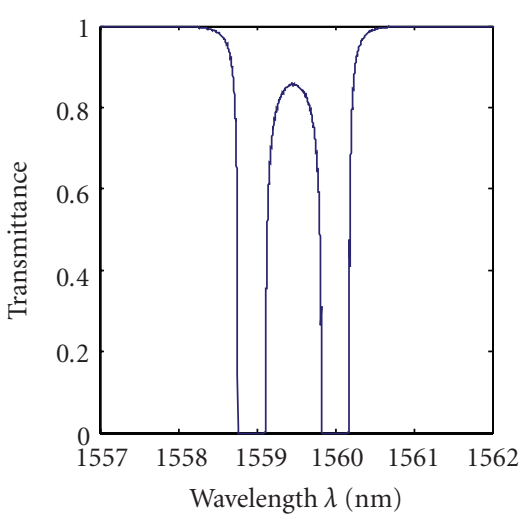

(a)

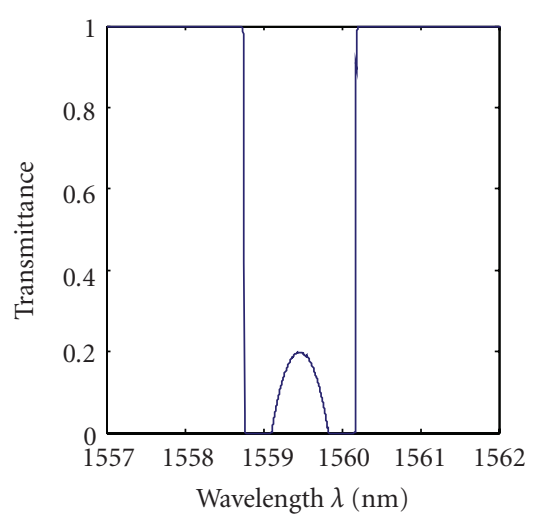

(b)

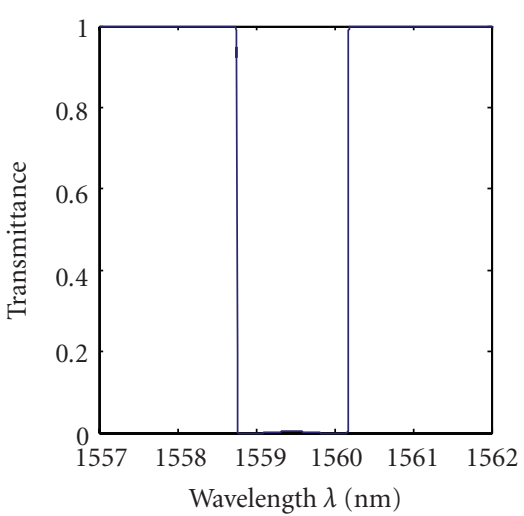

(c)

FigURE 3: Numerically computed spectral transmittance of FBGs used in numerical simulations of Figure 2 for $n_{0}=1.45, \Delta n=3.3 \times 10^{-4}$, and $\lambda_{B}=1560 \mathrm{~nm}$, corresponding to a grating length $L \simeq 24 \mathrm{~cm}$, and for increasing values of $l$ : (a) $l=0.1$, (b) $l=1.5$, and (c) $l=5$. 
experiment the tracing of pulse evolution in the grating (Figure 2) can be a nontrivial task, the signatures of KT can be simply obtained from standard spectral transmission measurements of the grating. In fact, for a given value of $\Delta V>2$ and according to the band diagram of Figure 1(a), in the KT regime a transmission window at $1<\Omega<\Delta V-1$ (the segment $\mathrm{AB}$ in Figure 1(a)), embedded into the two gaps $\Delta V-1<\Omega<\Delta V+1$ and $-1<\Omega<1$ (the segments $\mathrm{BC}$ and $\mathrm{AD}$ in Figure 1(a)) should be observed in the transmission spectrum, the suppression of $\mathrm{KT}$ for a smooth potential corresponding to the lowering of such a transmission window. This is clearly shown in Figure 3, where the spectral transmittance of the FBGs corresponding to the simulations of Figures 2(a), 2(b), and 2(c) are depicted. Note that, as the length $l$ of the chirped region is increased (from Figures 3(a) to 3(c)), the transmission window embedded in the two adjacent gaps disappears, which is the signature of KT inhibition.

\section{Conclusions}

In conclusion, a photonic analogue of Klein tunneling based on pulse propagation in nonuniform fiber Bragg gratings has been proposed. As compared to other photonic analogues of KT recently proposed in $[10,14]$ and based on spatial light propagation in periodic photonic structures, the phenomenon of KT in FBGs suggested in this work can be simply observed in the frequency domain as the opening of a transmission window inside the grating stop band, provided that the impressed chirp is realized over a length of the order of the analogue of the Compton wavelength. Such a FBGbased system might be thus considered to be the simplest optical analogue proposed so far to observe KT.

\section{Acknowledgment}

The author acknowledges financial support by the Italian MIUR (Grant no. PRIN-2008-YCAAK, Project "Analogie ottico-quantistiche in strutture fotoniche a guida d'onda").

\section{References}

[1] O. Klein, "Die Reflexion von Elektronen an einem Potentialsprung nach der relativistischen Dynamik von Dirac," Zeitschrift für Physik, vol. 53, no. 3-4, pp. 157-165, 1929.

[2] A. Calogeracos and N. Dombey, "History and physics of the Klein paradox," Contemporary Physics, vol. 40, no. 5, pp. 313321, 1999.

[3] F. Sauter, "Über das Verhalten eines Elektrons im homogenen elektrischen Feld nach der relativistischen Theorie Diracs," Zeitschrift für Physik, vol. 69, no. 11-12, pp. 742-764, 1931.

[4] P. Christillin and E. d'Emilio, "Role of the slope of realistic potential barriers in preventing relativistic tunneling in the Klein zone," Physical Review A, vol. 76, no. 4, Article ID 042104, 2007.

[5] A. Calogeracos, "Paradox in a pencil," Nature Physics, vol. 2, no. 9, pp. 579-580, 2006.

[6] M. I. Katsnelson, K. S. Novoselov, and A. K. Geim, "Chiral tunnelling and the Klein paradox in graphene," Nature Physics, vol. 2, no. 9, pp. 620-625, 2006.
[7] A. F. Young and P. Kim, "Quantum interference and Klein tunnelling in graphene heterojunctions," Nature Physics, vol. 5, no. 3, pp. 222-226, 2009.

[8] N. Stander, B. Huard, and D. Goldhaber-Gordon, "Evidence for Klein tunneling in graphene p-n junctions," Physical Review Letters, vol. 102, no. 2, Article ID 026807, 2009.

[9] S. Longhi, "Quantum-optical analogies using photonic structures," Laser and Photonics Reviews, vol. 3, no. 3, pp. 243-261, 2009.

[10] O. Bahat-Treidel, O. Peleg, M. Grobman, N. Shapira, T. PeregBarnea, and M. Segev, "Perfect Klein tunneling in anisotropic graphene-like photonic lattices," Physical Review Letters, vol. 104, Article ID 063901, 2010.

[11] R. A. Sepkhanov, Y. B. Bazaliy, and C. W. J. Beenakker, "Extremal transmission at the Dirac point of a photonic band structure," Physical Review A, vol. 75, no. 6, Article ID 063813, 2007.

[12] O. Peleg, G. Bartal, B. Freedman, O. Manela, M. Segev, and D. N. Christodoulides, "Conical diffraction and gap solitons in honeycomb photonic lattices," Physical Review Letters, vol. 98, no. 10, Article ID 103901, 2007.

[13] D. Ö. Güney and D. A. Meyer, "Negative refraction gives rise to the Klein paradox," Physical Review A, vol. 79, no. 6, Article ID 063834, 2009.

[14] S. Longhi, "Klein tunneling in binary photonic superlattices," Physical Review B, vol. 81, no. 7, 2010.

[15] J. Otterbach, R. G. Unanyan, and M. Fleischhauer, "Confining stationary light: dirac dynamics and Klein tunneling," Physical Review Letters, vol. 102, no. 6, Article ID 063602, 2009.

[16] A. M. Steinberg, P. G. Kwiat, and R. Y. Chiao, "Measurement of the single-photon tunneling time," Physical Review Letters, vol. 71, no. 5, pp. 708-711, 1993.

[17] S. Longhi, P. Laporta, M. Belmonte, and E. Recami, "Measurement of superluminal optical tunneling times in doublebarrier photonic band gaps," Physical Review E, vol. 65, no. 4, Article ID 046610, pp. 1-6, 2002.

[18] S. Longhi, M. Marano, M. Belmonte, and P. Laporta, "Superluminal pulse propagation in linear and nonlinear photonic grating structures," IEEE Journal on Selected Topics in Quantum Electronics, vol. 9, no. 1, pp. 4-16, 2003.

[19] J. E. Sipe, L. Poladian, and C. Martijn de Sterke, "Propagation through nonuniform grating structures," Journal of the Optical Society of America A, vol. 11, no. 4, pp. 1307-1320, 1994.

[20] L. Poladian, "Graphical and WKB analysis of nonuniform Bragg gratings," Physical Review E, vol. 48, no. 6, pp. 47584767, 1993.

[21] A. Asseh, H. Storøy, B. E. Sahlgren, S. Sandgren, and R. A. H. Stubbe, "A writing technique for long fiber Bagg gratings with complex reflectivity profiles," Journal of Lightwave Technology, vol. 15 , no. 8, pp. 1419-1423, 1997. 

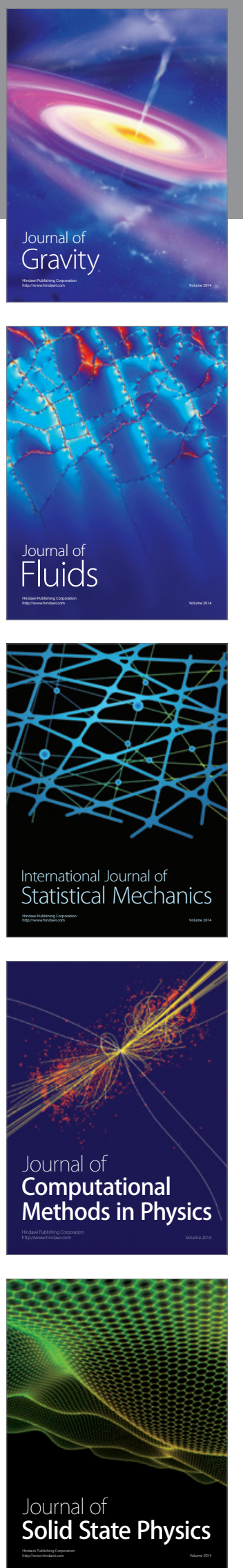

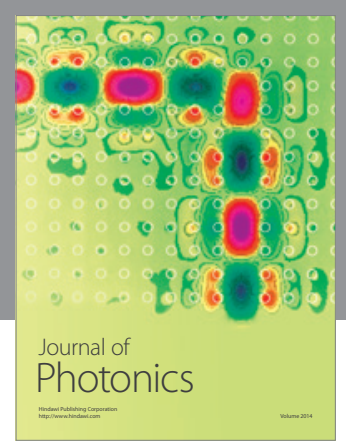

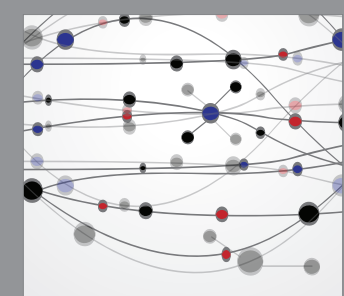

The Scientific World Journal
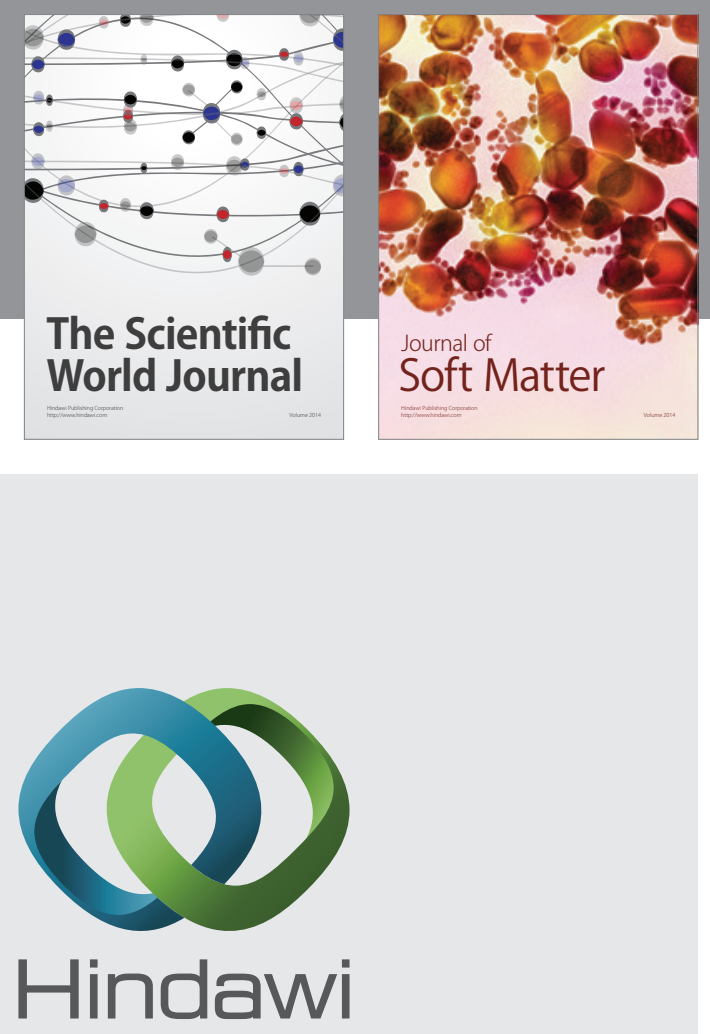

Submit your manuscripts at

http://www.hindawi.com
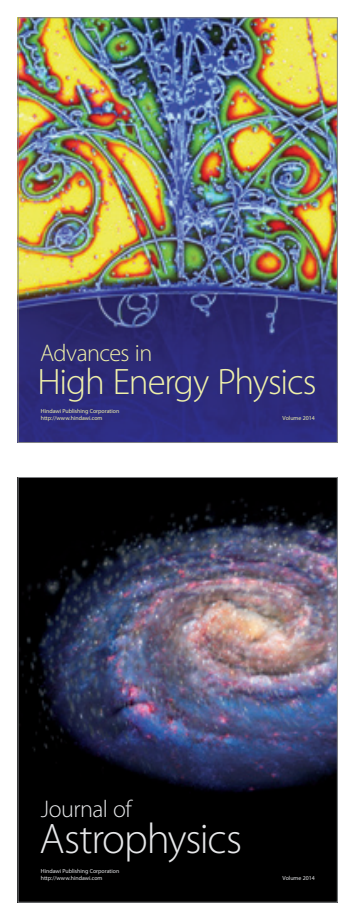
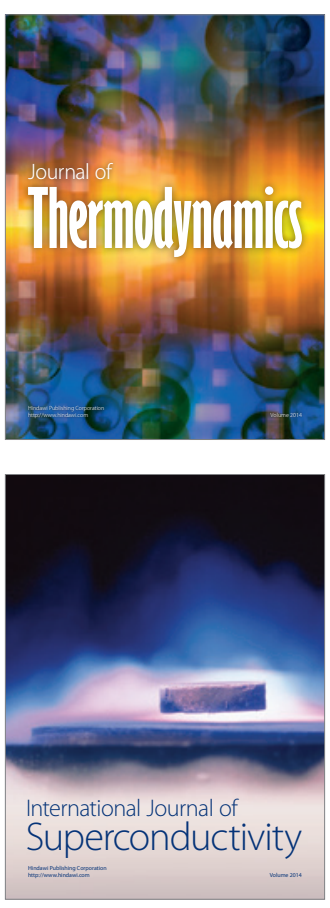
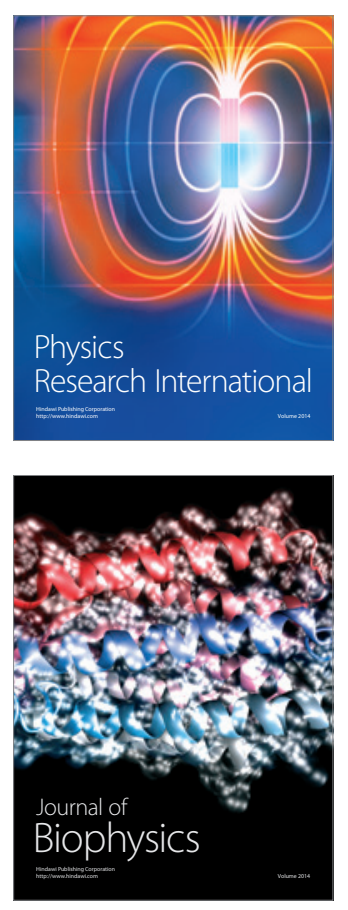
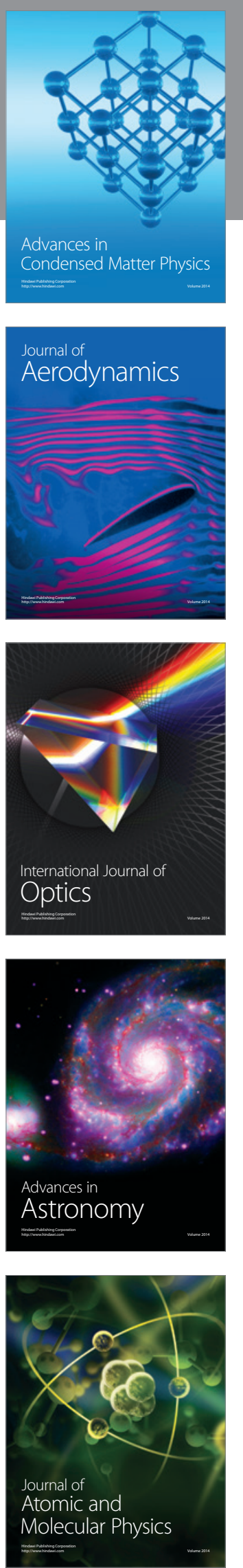